\title{
Associative Mechanosensory Conditioning of the Proboscis Extension Reflex in Honeybees
}

\author{
Martin Giurfa ${ }^{1,3}$ and Dagmar Malun ${ }^{2}$ \\ ${ }^{1}$ Centre de Recherches sur la Cognition Animale, CNRS-Université Paul-Sabatier, 31062 Toulouse cedex 4, France; \\ ${ }^{2}$ Neurobiology, Institute of Biology, Free University of Berlin, D-14195 Berlin, Germany
}

\begin{abstract}
The present work introduces a form of associative mechanosensory conditioning of the proboscis extension reflex (PER) in honeybees. In our paradigm, harnessed honeybees learn the elemental association between mechanosensory, antennal stimulation and a reward of sucrose solution delivered to the proboscis. Thereafter, bees extend their proboscis to the antennal mechanosensory stimulation alone. We show that bees can learn such an association in a side-specific manner, that is, they learn the association on the antennal side that was rewarded and not on the side that was not rewarded. Responding produced by the paired training does likely contain a substantial Pavlovian component. Responding is only elicited by mechanosensory stimulation and not by spurious cues such as olfactory, visual, and contextual ones. The interstimulus interval (ISI) affects one-trial mechanosensory learning: a bell-shaped curve with a maximum of responding $\sim 4 \mathrm{sec}$ ISI was obtained. Mechanosensory memory is still operative $24 \mathrm{~h}$ after conditioning. Apart from absolute conditioning in which mechanosensory stimulation of one antenna is paired with sucrose, differential, side-specific, mechanosensory conditioning using two mechanosensory stimulations, one rewarded and the other not, is also possible. This paradigm constitutes, therefore, a new standard procedure for further learning studies in honeybees.
\end{abstract}

Associative learning is a fundamental property of nervous systems governed by rules applicable both across species and across modalities. In classical conditioning (Pavlov 1927), animals learn that an originally neutral stimulus (conditioned stimulus, CS) can act as a predictor for a biologically significant stimulus (unconditioned stimulus, US). In operant conditioning (Skinner 1938), animals learn that a given response (operant action) is followed by the occurrence or suppression of a given reinforcement.

The honeybee, Apis mellifera, constitutes a traditional model for the study of learning and memory at the behavioral, cellular, and molecular level (Menzel et al. 1993; Hammer and Menzel 1995; Menzel and Müller 1996; Menzel 1999; Menzel and Giurfa 2001; Giurfa 2003a,b). In a natural context, honeybees learn and memorize the local cues that characterize places of interest such as the hive and flowers in the surroundings. In the laboratory, the bees' learning abilities are mainly studied using a wellestablished paradigm: the olfactory conditioning of the proboscis extension reflex (PER; Takeda 1961; Bitterman et al. 1983). When the main chemosensory organs of a hungry bee, the antennae, are touched with sucrose solution, the insect reflexively extends its proboscis (PER) to reach out toward the sucrose and lick it. Bees immobilized in individual harnesses learn to associate an odor (conditioned stimulus or CS) with a reward of sucrose solution (unconditioned stimulus or US) delivered to their antennae and to the proboscis. Thus, they learn to extend their proboscis to the mere presentation of the odor. This effect is clearly associative and involves classical and not operant conditioning (Bitterman et al. 1983) as shown by the omission procedure, in which the occurrence of the conditioned response (the extension of the proboscis) prevents occurrence of the US (Bitterman et al. 1983). Bees trained in this way learn to respond to the odor

\section{${ }^{3}$ Corresponding author.}

E-MAIL giurfa@cict.fr; FAX 33561556154.

Article and publication are at http://www.learnmem.org/cgi/doi/10.1101/ Im.63604. despite the omission training, thus showing that the association learned is classical and not operant.

Interestingly, beside olfactory PER conditioning, few laboratory paradigms exist allowing the study of honeybee learning and memory under controlled conditions. Excluding the different variants that use free-flying (or free-walking) bees (see Giurfa 2003a) in which a precise control of the timing of the conditioned stimuli is difficult, very few alternatives exist to olfactory PER conditioning. Usually these combine olfactory stimulation with an additional CS (Pelz et al. 1997; Gerber and Smith 1998). Nevertheless, using bees immobilized in individual harnesses, Erber and coworkers developed an antennal tactile learning paradigm, which has proven to be extremely useful to address questions going from individual learning (Erber et al. 1997, 1998; Kisch and Erber 1999) to genotype, foraging role, and sucrose responsiveness specializations (Scheiner et al. 2001a). This paradigm admits three basic variants: In the first one, nonassociative learning is involved as the bee learns the contour of an object placed close to its head without being rewarded, such that it can reproduce the contour afterward even if the object is absent (Erber et al. 1997). In the second one, operant learning is involved as the bee is rewarded when its frequency of antennal contacts with an object (a plate close to the bee's head) exceeds a certain threshold (Kisch and Erber 1999). As a result of this conditioning, bees increase their frequency of antennal contacts with the reinforced object. In the third variant, bees are reinforced after scanning the surface of a given object with their antennae to learn its texture properties (Erber et al. 1998; Scheiner et al. 1999, 2001a). In this case, the associations established are difficult to characterize with respect to their operant or classical nature. In all three cases, however, learning depends on the operant activity of the bee during antennal movements.

The advantages provided by this conditioning paradigm as well as by olfactory PER conditioning are related to the fact that bees are restrained and therefore cannot move. Both paradigms allow studying the rules underlying associative and nonassociative learning (Bitterman et al. 1983; Menzel and Bitterman 1983; 
Erber et al. 1997, 1998; Kisch and Erber 1999) and the processing of sensory stimuli (Chandra and Smith 1998; Erber et al. 1998; Deisig et al. 2001, 2002, 2003). Secondly, they allow studying the physiological correlates of learning at different levels, ranging from the molecular and biochemical one to that of single identified neurons and neuronal ensembles (Hammer and Menzel 1995; Menzel and Müller 1996; Menzel 1999; Erber et al. 2000). These studies are possible because immobilized bees still perform and learn after their brains are exposed. It is thus possible to perform pharmacological, electrophysiological, and optical imaging experiments in parallel with conditioning to understand the neurobiological basis of associative learning (Pribbenow and Erber 1996; Faber et al. 1999; Erber et al. 2000; Cano Lozano et al. 2001; Sandoz et al. 2003).

In the present work, we introduce a different conditioning paradigm of the proboscis extension reflex. Such a paradigm allows for controlled side-specific learning, which is, in principle, also possible in the case of olfactory learning (Masuhr and Menzel 1972; Sandoz and Menzel 2001; Sandoz et al. 2002) by placing and fixating a separating wall between the two antennae to avoid cross-stimulation with the conditioned odors. The paradigm studied here is the "mechanosensory conditioning of the PER." In such a paradigm, harnessed bees have to learn an association between a mechanosensory stimulation of one antenna (CS: gentle stimulation of one antenna with a rod) and a reward of sucrose solution (US) delivered to the proboscis of the animal. The use of mechanosensory stimuli for conditioning PER has been reported before (Pelz et al. 1997; Giurfa et al. 2001; Menzel et al. 2001) but has never been appropriately characterized to standardize it for regular use in the laboratory. Different from previous studies on tactile antennal learning (Erber et al. 1997, 1998; Kisch and Erber 1999), the paradigm presented here contains substantial Pavlovian components. Specifically, we raised the question of whether it is possible to condition PER in an associative way using mechanosensory stimulation as conditioned stimulus. We asked whether one-sided mechanosensory stimulation of the antennae could be associated with a reward of sucrose solution such that the mechanosensory stimulation alone is able to elicit PER in a side-specific manner. We determined the optimal interstimulus interval in this paradigm and analyzed whether mechanosensory memory is stable and allows for transfer between sides. Finally, in differential conditioning, we studied whether the two antennae can learn to discriminate two separate mechanosensory stimulations, one rewarded and the other not.

\section{RESULTS}

\section{Absolute Mechanosensory Conditioning}

In absolute mechanosensory conditioning, mechanosensory stimulation on one antenna was paired with a reward of sucrose solution.

\section{First Experiment}

Two independent subgroups of 40 bees were trained to associate a mechanosensory stimulation provided by a toothpick contact on one antenna (CS) with a reinforcement of sucrose solution (US; Fig. 1). One subgroup was conditioned on the right antenna and the other on the left antenna. Conditioning proceeded along six explicitly paired trials.

There were no significant differences between subgroups $\left(F_{1,78}=0.31 ; \mathrm{NS}\right)$ and the interaction was nonsignificant $\left(F_{5,390}=0.06\right.$; NS $)$, thus indicating that acquisition was the same for both the left-conditioned and the right-conditioned subgroup. Therefore, the data of both subgroups were pooled and are presented in Figure 2. This figure shows the response of bees (\%

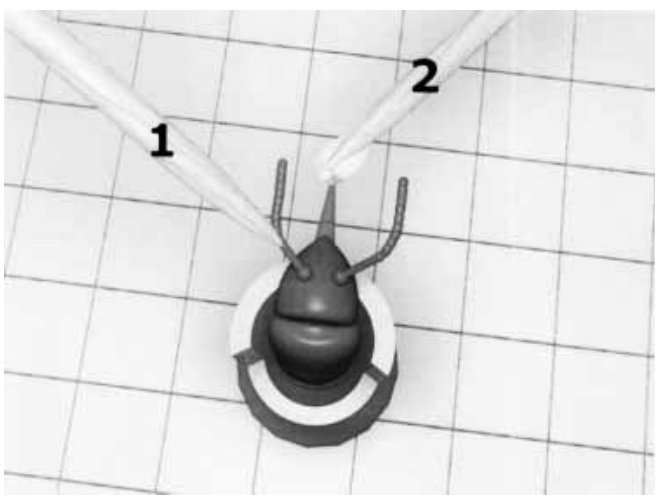

Figure 1 Overall view of the setup used for mechanosensory conditioning. A honeybee was placed in a holder. Mechanosensory stimulation provided by a toothpick (1) was applied to one antenna, whereas the proboscis was rewarded with sucrose solution (2). Thus, the bee was trained to associate the mechanosensory stimulation of one antenna (CS) with the reinforcement of sucrose solution (US).

PER) along the six conditioning trials. The probability of PER significantly increased along trials $\left(F_{5,395}=50.74 ; P<0.0001\right)$. Acquisition was rapid with asymptotic performance reached by the third trial, thus indicating that bees can learn an association between a mechanosensory stimulation and sucrose solution.

\section{Second Experiment}

The performance of bees trained with explicitly paired presentations of the CS and the US was compared with that of bees trained with explicitly unpaired presentations of the CS and the US. Four subgroups of 21 bees were used in this experiment. Two of them received explicit CS-US pairing (one subgroup conditioned on the left and the other on the right antenna); they were given six CS-US trials alternated with six blank trials in a pseudorandom sequence. During a blank trial, the bee was placed in the experimental position and no specific stimulus was delivered. The other two subgroups received unpaired CS-only and US-only presentations (one subgroup received the CS on the left and the other on the right antenna); they were given six CS-only and six US-only trials presented in a pseudorandom sequence. The function of the blank trials for the explicitly paired group was to equate the number of placements between both groups.

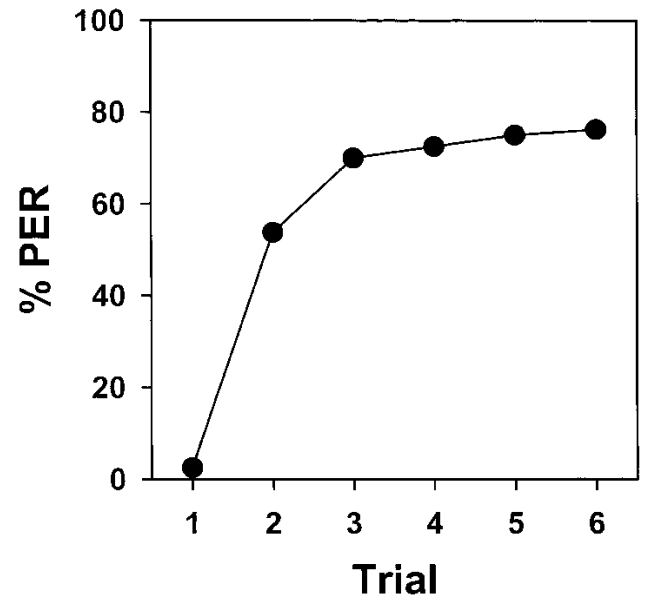

Figure 2 Mechanosensory antennal conditioning of the proboscis extension reflex (\% PER) of bees along six training trials. The probability of PER significantly increased along training. 


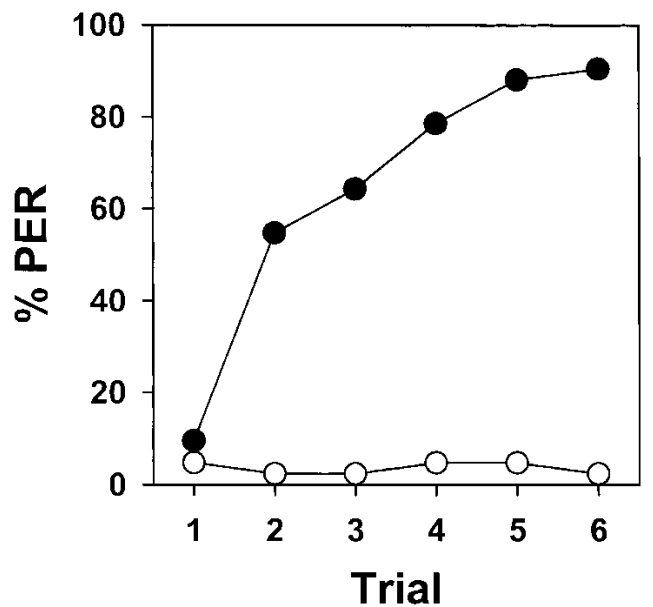

Figure 3 Associative nature of mechanosensory antennal conditioning of PER. Bees were trained either with explicitly paired presentations of the CS and the US (filled circles) or with explicitly unpaired presentations of the CS and the US (empty circles). The probability of PER significantly increased only for the explicitly paired group, thus showing that responding produced by the paired training had a true associative basis and was not merely due to experience with the stimuli independently of their temporal relationship.

The two subgroups of bees trained with paired CS-US presentations did not differ significantly from each other $\left(F_{1,40}=0.20 ; \mathrm{NS}\right)$ and the interaction was also not significant $\left(F_{5,200}=0.64 ; \mathrm{NS}\right)$; their data were therefore pooled. The resulting curve is shown in Figure 3 (filled circles). Bees showed rapid acquisition $\left(F_{5,205}=29.84 ; P<0.0001\right)$ with asymptotic performance reached by the fourth trial. The two subgroups of bees conditioned with the unpaired CS-only, US-only presentations did not differ significantly from each other $\left(F_{1,40}=0.65 ; \mathrm{NS}\right)$, and the interaction was also not significant $\left(F_{5,200}=1.13\right.$; NS); their data were therefore also pooled. The resulting curve is also shown in Figure 3 (empty circles) and shows negligible response and no significant variation along trials $\left(F_{5,205}=0.59\right.$; NS). This curve significantly differed from that obtained for the explicitly paired group $\left(F_{1,82}=207.41 ; P<0.00001\right)$, thus showing that the responses produced by the paired training had, indeed, a true associative basis and were not merely due to experience with the stimuli independently of their temporal relationship.

\section{Third Experiment}

To discern whether bees associated the mechanosensory stimulus (CS) with the reward of sucrose solution (US; classicalPavlovian-conditioning), or the act of extending their proboscis (response) with the reinforcement of sucrose solution (US; operant conditioning), two groups of bees, omission and yoked controls, were conditioned in a coupled way. Two subgroups of 21 bees were conditioned following an "omission procedure" along six trials. In other words, six CS-US presentations were given to the bees, which were rewarded only when they did not extend their proboscis to the mechanosensory stimulation of one antenna, that is, the occurrence of the conditioned response prevented the occurrence of the US. In these cases, bees were given a toothpick imbibed with sucrose solution directly to the mouthparts, which elicited proboscis extension reflex. One subgroup was stimulated on the right and the other on the left antenna. Two other subgroups of 21 bees were trained as "yoked controls": Each bee was given the reinforcement sequence of a corresponding bee of the omission subgroup whatever its response to the CS; one subgroup was stimulated on the right and the other on the left antenna.
There were no significant differences between the two omission subgroups, thus showing that the antenna chosen for mechanosensory stimulation was not relevant $\left(F_{1,40}=1.91 ; \mathrm{NS}\right)$. The interaction was also not significant $\left(F_{5,200}=0.25\right.$; NS) such that both subgroups were pooled. Figure 4 shows the acquisition of the bees trained under omission procedure (filled circles). Bees significantly increased their response to the mechanosensory stimulation along trials $\left(F_{5,205}=29.29 ; P<0.00001\right)$. The fact that acquisition occurred despite suppression of the US whenever the conditioned response occurred suggests that associations established in the normal mechanosensory conditioning procedure are rather Pavlovian than operant. This was verified by analyzing the performance of the yoked subgroups.

The two yoked subgroups (left and right antenna) did not differ significantly $\left(F_{1,40}=0.44 ; \mathrm{NS}\right)$ and the interaction was also not significant $\left(F_{5,200}=0.47 ; \mathrm{NS}\right)$. Both subgroups were therefore pooled. Figure 4 shows the acquisition of the yoked control (empty circles). Bees significantly increased their response to the mechanosensory stimulation along trials $\left(F_{5,205}=33.73\right.$; $P<0.00001)$. Both the omission and the yoked group showed the same acquisition $\left(F_{1,82}=0.05 ; \mathrm{NS}\right)$, and the interaction was not significant $\left(F_{5,410}=0.30\right.$; NS). This result indicates that the association established in this paradigm is Pavlovian and not operant.

This conclusion could, however, be challenged because a residual operant component may subsist in the yoked bees. In the six conditioning trials, the omission bees were rewarded whenever they did not show PER, that is, in $2.8 \pm 0.04$ trials (mean $\pm \mathrm{SE} ; n=42$ ). The yoked bees, assigned to the omission bees, extended their proboscis in $0.8 \pm 0.03$ trials of these 2.8 trials (mean $\pm \mathrm{SE} ; n=42$ ). It could be thus argued that in 0.8 trials, an association between response and US might be established in addition to the CS-US association, and that the differential (occasions of US presentations) between the two groups, omission and yoked, is too small to be detected. It is therefore worth assuming a cautious viewpoint and concluding that our mechanosensory conditioning paradigm does likely contain a substantial Pavlovian component. However, more extensive research using prolonged training and a weaker US (Brembs and Heisenberg 2000) is necessary to exclude definitively any operant contribution.

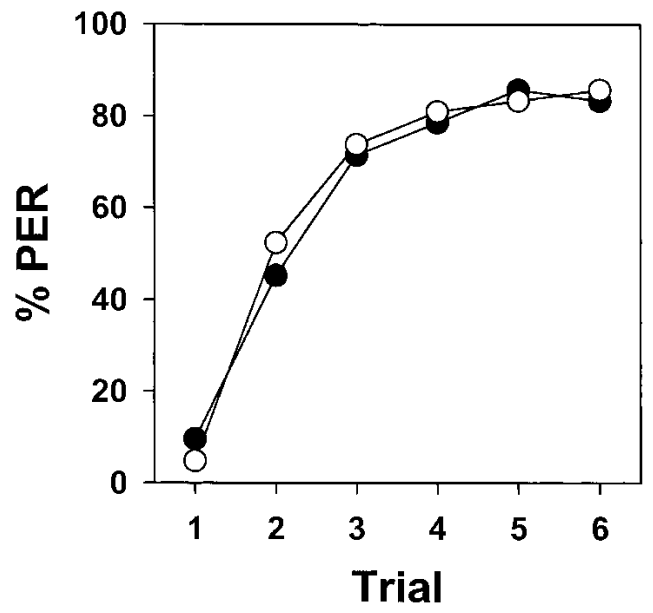

Figure 4 Test of the classical nature of mechanosensory antennal condition of PER. Bees were trained following an omission procedure (filled circles) or as yoked controls (empty circles). Bees of both groups equally increased their response significantly to the mechanosensory stimulation along the six training trials. This result shows that the association established during conditioning is independent of the response (extension of the proboscis) of the bees and is therefore classical and not operant. 


\section{Fourth Experiment}

This experiment was conceived as a control for spurious stimulations that might act as conditioned stimuli beyond mechanosensory contact. First, we wanted to know whether olfactory information present on the toothpick (e.g., wood odor) was used as the CS in this paradigm. The insect antenna is a complex sensory organ presenting different types of sensory receptors such that it is difficult to determine the real nature of a mechanosensory stimulation. The contribution of olfactory information has to be determined to make sure that true mechanosensory conditioning occurs. To this end, we performed a transfer experiment in which bees were conditioned in the traditional manner (stimulation provided by the toothpick) and afterward tested with a glass capillary. Two subgroups of 20 bees were conditioned along six explicitly paired trials, one on the right antenna and the other on the left antenna. In both groups, the probability of PER significantly increased along acquisition trials $\left(F_{5,190}=53.99\right.$; $P<0.0001)$.

As there were no significant differences between both groups $\left(F_{1,38}=0.10 ; \mathrm{NS}\right)$, their performance was pooled and a single acquisition curve was obtained (Fig. 5A, filled circles). Then, $10 \mathrm{~min}$ after the last acquisition trial, four retention tests were performed spaced by $10 \mathrm{~min}$ and in a random sequence (Fig. $5 \mathrm{~B}$, black bars): in two of them (ipsilateral and contralateral; i.e., stimulation of the trained and of the untrained antenna, respectively) a toothpick was used to stimulate the antenna as it was done during acquisition (Fig. 5B; IT, ipsilateral toothpick; CT, contralateral toothpick); in the other two tests (ipsilateral and contralateral), the toothpick was replaced by a glass capillary (Fig. 5B; IG, ipsilateral glass; CG, contralateral glass). The response of

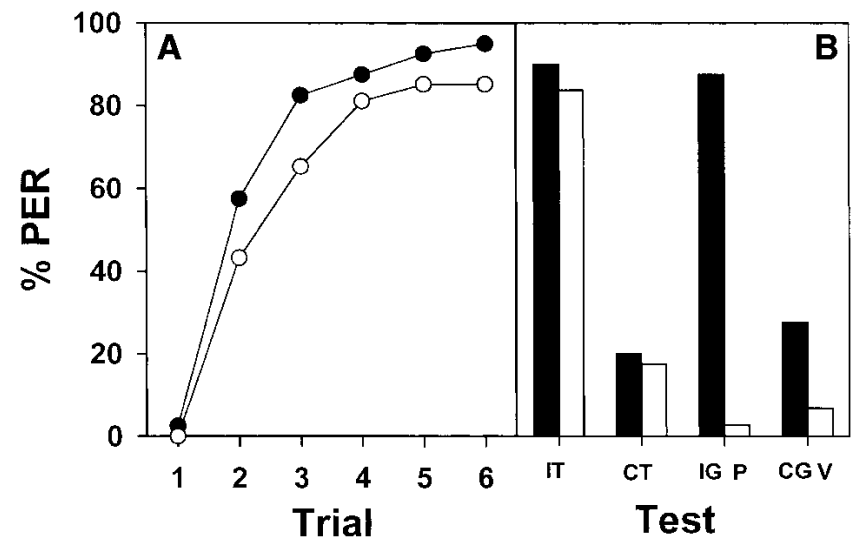

Figure 5 Control of spurious stimulations in mechanosensory antennal conditioning of honeybees. One group of bees was used to test whether olfactory information present on the toothpick (see Fig. 1) was used in our paradigm (filled circles, black bars). Another group was used to test the incidence of visual and contextual cues in the response of conditioned animals (empty circles, white bars). ( $A$ ) Both groups were trained as usual and learned to react with PER to the mechanosensory stimulation of one antenna. (B) Both groups received four extinction tests. One group (black bars) was tested with ipsilateral (I) and contralateral ( $C$; relative to the antenna used for conditioning) antennal stimulation using either the toothpick $(T)$ or a clean glass capillary $(G)$. This group was thus tested with the following tests in a random sequence: IT, CT, IG, CG. Bees responded equally well to the antennal stimulation with the glass capillary, thus showing that olfactory cues from the toothpick were not relevant. The other group (white bars) was tested with ipsilateral (I) and contralateral ( $C$; relative to the antenna used for conditioning) antennal stimulation using the toothpick $(T)$, with a simple placement in the experimental place $(P)$ and with movements of the toothpick close to the antenna without contacting it $(\mathrm{V})$. This group was thus tested with the following tests in a random sequence: IT, CT, P, V. Bees did not respond significantly to contextual $(P)$ or to visual $(V)$ cues. bees did not vary depending on the stimulated antenna (left or right; $F_{1,38}=0.00$; NS). The data were therefore grouped and discriminated only with respect to the kind of material used for stimulation (toothpick vs. glass capillary) and the side-specific conditioning (ipsilateral vs. contralateral; Fig. 5B, black bars). The response of the bees varied significantly depending on the retention test $\left(F_{3,117}=34.99 ; P<0.0001\right)$. The response to the ipsilateral stimulation was the same for both the toothpick and the glass capillary $\left(F_{1,39}=0.11 ; \mathrm{NS}\right)$. In the same way, the response to the contralateral stimulation was the same for both the toothpick and the glass capillary $\left(F_{1,39}=0.59 ; \mathrm{NS}\right)$. Thus, bees responded equally well to the toothpick and the glass capillary. The only significant difference was between ipsilateral and contralateral stimulations $\left(F_{1,79}=101.92 ; P<0.0001\right)$ independently of the material used for the mechanosensory stimulation.

Secondly, we wanted to know whether beyond mechanosensory stimulation, visual and/or contextual information was used as the CS in this paradigm. Visual stimulation was provided by placing the toothpick in a similar position as in mechanosensory conditioning but without contacting the antenna. Contextual stimulation was provided by placing the bees in the conditioning position without further stimulation ("placement"). Two groups of 37 bees were conditioned along six explicitly paired trials, one on the right antenna and the other on the left antenna. Again, the probability of PER significantly increased along acquisition trials $\left(F_{5,360}=76.67 ; P<0.0001\right)$, and there were no significant differences between groups $\left(F_{1,72}=0.31\right.$; NS). Therefore, the performance of both groups was pooled and a single acquisition curve was obtained (Fig. 5A, empty circles). Then, 10 min after the last acquisition trial, four retention tests were performed spaced by $10 \mathrm{~min}$ and in a random sequence (Fig. 5B, white bars): mechanosensory ipsilateral (IT) and contralateral stimulation $(\mathrm{CT})$, placement $(\mathrm{P})$ and visual ipsilateral $(\mathrm{V})$. The response of bees did not vary depending on the antenna stimulated (left or right; $F_{1,72}=0.82$; NS) but changed significantly with the type of retention test performed $\left(F_{3,216}=101.79\right.$; $P<0.0001)$. The data were therefore grouped and discriminated only with respect to the four kinds of test performed. Bees again responded preferentially to the ipsilateral mechanical stimulation (ipsi vs. contra: $F_{1,73}=175.02 ; P<0.0001$ ). Moreover, their response to the stimulation provided by the placement alone (Fig. 5B, P) was identical to that provided by the movement of the toothpick (Fig. 5B, V), close to the ipsilateral antenna (placement vs. visual: $F_{1,73}=1.29$; NS). In both cases, bees did not react significantly to these stimulations. Thus, we confirmed that, as a result of the six acquisition trials, bees learned to associate the reinforcement of sucrose solution with the mechanosensory stimulation of the antenna, and not with spurious stimuli of the environment.

\section{Fifth Experiment}

Knowing that bees in this paradigm learn to associate mechanosensory stimulation with a reinforcement of sucrose solution, we determined the optimal interstimulus interval (ISI) for such a conditioning. The ISI constitutes a determinant feature in Pavlovian conditioning. We trained 12 independent groups of 20 bees with a single mechanosensory stimulation $(4 \mathrm{sec})$ paired with a reward of sucrose solution $(3 \mathrm{sec})$. We then measured, for each group, the response to a second mechanosensory stimulation (one-trial learning). The retention test was performed 10 min after the learning session. Two groups received backward conditioning (i.e., the US preceded the CS; group 1: ISI $=-3 \mathrm{sec}$ ) with the CS either on the left (group 1) or the right (group 2) antenna; two groups received simultaneous presentation of CS and US (group 2: ISI = $0 \mathrm{sec}$ ) with the CS either on the left (group 3 ) or the right (group 4) antenna; and eight groups received for- 
ward conditioning (i.e., the CS preceded the US; groups 5 and 6: ISI = 3 sec; groups 7 and 8: ISI $=4 \mathrm{sec}$; groups 9 and 10: ISI $=8 \mathrm{sec}$; and groups 11 and 12: ISI = $12 \mathrm{sec}$ ) with the CS either on the left (groups 5, 7, 9, and 11) or on the right (group 6, 8, 10, and 12) antenna.

Within each ISI, the performance of both groups (left and right conditioned) did not differ such that results were pooled (groups 1 vs. 2 : $F_{1,38}=0.35$; NS; groups 3 vs. $4: F_{1,38}=0.11$; NS; groups 5 vs. $6: F_{1,38}=0.97$; NS; groups 7 vs. 8: $F_{1,38}=0.10$; NS; groups 9 vs. $10: F_{1,38}=0.42$; NS; groups 11 vs. $12: F_{1,38}=0.17$; NS). Figure 6 shows the response of bees to the second mechanosensory stimulation as depending on ISI. The different ISI groups differed significantly in their response to the CS after a single learning trial, thus showing that ISI affects mechanosensory acquisition $\left(F_{5,34}=9.57 ; P<0.0001\right)$. The ISI curve showed the typical bell-shaped form found in classical conditioning, with a maximum around 3 4 sec ISI. Backward procedures did not yield significant acquisition, whereas forward procedures did.

\section{Sixth Experiment}

We analyzed whether mechanosensory memory is stable and allows transfer between sides. Two subgroups of 30 bees were conditioned with six explicitly CS-US paired presentations. One subgroup was conditioned on the right antenna and the other on the left antenna. Conditioning proceeded along six explicitly paired trials. Bees learned rapidly to associate the mechanosensory stimulation with the sucrose reward. There were no significant differences between subgroups $\left(F_{1,58}=0.05\right.$; NS) and the interaction was nonsignificant $\left(F_{5,290}=0.15\right.$; NS). Thus, both acquisition curves were pooled (Fig. 7A, empty circles).

Retention tests were done $1 \mathrm{~min}, 10 \mathrm{~min}, 1 \mathrm{~h}$, and $24 \mathrm{~h}$ after the last acquisition trial. In each retention test, bees were presented with mechanosensory ipsilateral and contralateral stimulation. Ipsilateral and contralateral stimulations followed a random sequence. In all tests, there were no differences between the group conditioned on the left antenna and that conditioned on the right antenna ( $1 \mathrm{~min}: F_{1,58}=0.33$; NS; $10 \mathrm{~min}: F_{1,58}=0.05$;



Figure 6 Determination of the optimal interstimulus interval (ISI) for mechanosensory conditioning in bees via one-trial-learning. Different groups of bees were given a single mechanosensory antennal stimulation (CS: $4 \mathrm{sec}$ ) paired with a reward of sucrose solution (US: $3 \mathrm{sec}$ ). Subsequently, the response to a second mechanosensory stimulation was measured. The intertrial interval was $10 \mathrm{~min}$. Six different ISIs were tested: -3 sec (backward conditioning: the US precedes the CS), $0 \mathrm{sec}, 3 \mathrm{sec}, 4 \mathrm{sec}$, $8 \mathrm{sec}$, and $12 \mathrm{sec}$ (forward conditioning: the CS precedes the US). The optimum ISI lies around 3-4 sec.

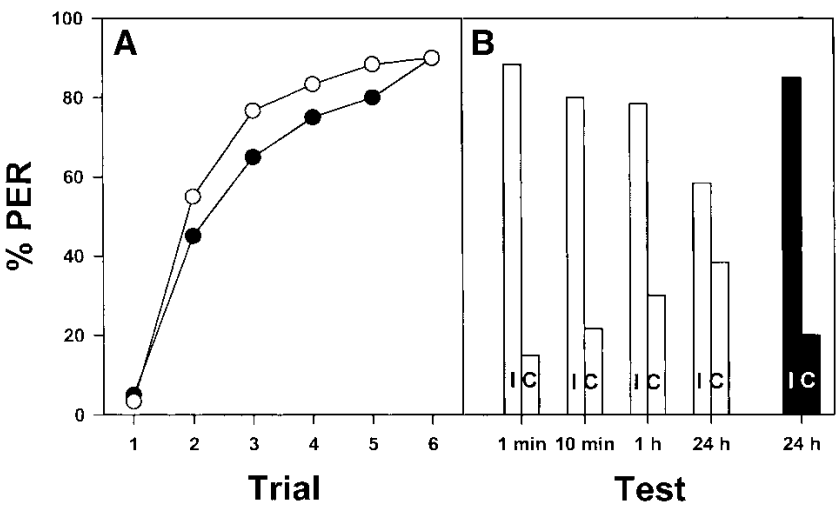

Figure 7 Retention tests after mechanosensory, antennal conditioning of PER. (A) Bees were conditioned as usual. During acquisition, the probability of PER significantly increased during the six CS-US paired presentations. (B) Retention tests were performed at $1 \mathrm{~min}, 10 \mathrm{~min}, 1 \mathrm{~h}$, and $24 \mathrm{~h}$ after the last acquisition trial. In such tests, bees received an ipsilateral (I) and a contralateral (C) antennal stimulation (with respect to the antenna previously conditioned). In all cases, bees responded always significantly more to the ipsilateral than to the contralateral stimulation.

NS; 1 h: $F_{1,58}=0.33$; NS; 24 h: $F_{1,58}=0.12$; NS). Therefore, results were pooled and discriminated according to the kind of stimulation provided in the test (ipsilateral vs. contralateral) and the time of the retention test ( $1 \mathrm{~min}, 10 \mathrm{~min}, 1 \mathrm{~h}, 24 \mathrm{~h}$; Fig. 7B, empty bars).

There were no significant differences between retention times $\left(F_{3,236}=0.36\right.$; NS), but in all tests, bees responded significantly more to the ipsilateral than to the contralateral stimulation $\left(F_{1,236}=179.48 ; P<0.00001\right)$. The interaction was also significant $\left(F_{3,236}=9.07 ; P<0.0001\right)$. This significance is due to different patterns of response depending on the test. Note that the probability of responding to the ipsilateral stimulation decreased from $88 \%$ to $58 \%$ and that the probability of responding to the contralateral stimulation varied between $15 \%$ and $38 \%$ from the first $(1 \mathrm{~min})$ to the fourth $(24 \mathrm{~h})$ test. These results can be caused by the repeated testing of the same group of bees. As no reward was provided during the tests, testing may result in extinction of the ipsilateral response and in enhanced generalization between both ipsi and contralateral sides as bees may learn that both kinds of stimulations, ipsi and contralateral, have the same consequence, namely, no US delivery.

To test this possibility, we trained another group of bees in the same way, but subjected them to a single test, $24 \mathrm{~h}$ after the last acquisition trial. Performance in this unique test can thus be compared with that in the test at $24 \mathrm{~h}$ of bees that were repeatedly tested. Two subgroups of 10 bees were conditioned with six explicitly CS-US paired presentations. One subgroup was conditioned on the right antenna and the other on the left antenna. There were no significant differences between subgroups $\left(F_{1,18}=0.38 ; \mathrm{NS}\right)$ and the interaction was nonsignificant $\left(F_{5,90}=0.39 ; \mathrm{NS}\right)$. Thus, both acquisition curves were pooled (Fig. $7 \mathrm{~A}$, filled circles)

In the test at $24 \mathrm{~h}$, there were no differences between the group conditioned on the left antenna and that conditioned on the right antenna $\left(F_{1,18}=0.18\right.$; NS). Therefore, results were pooled and discriminated according to the side of stimulation (Fig. 7B, filled bars). In this test, bees showed a discrimination performance that was comparable at that of the test at $1 \mathrm{~min}$ of the group repeatedly tested. Thus, $24 \mathrm{~h}$ after conditioning, bees are perfectly capable of distinguishing the conditioned from the nonconditioned side. The decay observed in the group of bees repeatedly tested may be caused by the additive effects of extinction and enhanced generalization. 


\section{Differential Mechanosensory Conditioning}

\section{Seventh Experiment}

Differential conditioning constitutes a within-group control procedure that allows verifying whether side-specific mechanosensory learning is indeed possible and based on an associative effect rather than on exposure to the training stimuli independently of their reinforcement history. Two subgroups of 41 bees were conditioned along 12 acquisition trials: six trials associated mechanical stimulation on one antenna (left or right) with sucrose solution (positive trials) and six trials associated mechanical stimulation on the alternative antenna (right or left) with absence of reward (negative trials). Groups were therefore termed $(\mathrm{R}+/ \mathrm{L}-)$ and $(\mathrm{R}-/ \mathrm{L}+)$. Positive and negative trials were alternated in a pseudorandom sequence, as it is usually done in olfactory differential conditioning.

There were no differences between both groups $\left(F_{1,160}=0\right.$; NS) such that results were pooled. Figure 8A shows the acquisition in terms of responses to the reinforced (filled circles) and to the nonreinforced stimuli (empty circles). Both curves differed significantly from each other $\left(F_{1,162}=226.26 ; P<0.00001\right)$, and the interaction was also significant $\left(F_{5,810}=68.02 ; P<0.00001\right)$. The curve showing the probability of responding to the reinforced stimulus increased significantly along the six positive trials $\left(F_{5,405}=74.40 ; P<0.0001\right)$, whereas that showing the probability of responding to the negative stimulus decreased significantly along the six negative trials $\left(F_{5,405}=7.92 ; P<0.0001\right)$.

Then, $10 \mathrm{~min}$ after the last acquisition trial, two retention tests were performed spaced by $10 \mathrm{~min}$ and in a random sequence to determine the response to ipsilateral (stimulation of the antenna previously reinforced with sucrose solution) and contralateral (stimulation of the antenna stimulated but nonreinforced) stimulation. The responses of both groups $(\mathrm{R}+/ \mathrm{L}-$ and $\mathrm{R}-/ \mathrm{L}+)$ did not differ in the retention tests $\left(F_{1,80}=0.22\right.$; NS $)$ such that they were pooled and presented according to ipsilateral and contralateral stimulation (Fig. 8B). The responses to the ipsilateral mechanosensory stimulation (filled bar) were significantly

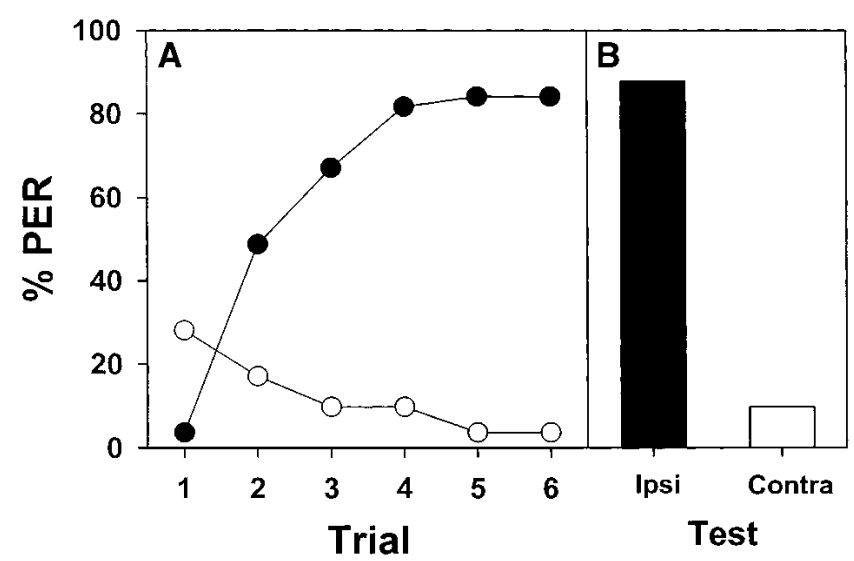

Figure 8 Side-specific, differential, antennal mechanosensory conditioning of PER. (A) During acquisition, mechanical stimulation of one antenna was paired with the sucrose solution reward (CS+ trials: filled circles), whereas mechanical stimulation of the other antenna was not (CS - : empty circles). Bees were conditioned along 12 trials (six CS + and six CS - trials) in a pseudorandom sequence. The probability of responding to the CS+ increased significantly, whereas the probability of responding to the CS - decreased significantly during acquisition. (B) Retention tests were performed $10 \mathrm{~min}$ after the last acquisition trials. Bees received an ipsilateral (I) and a contralateral (C) antennal stimulation (with respect to the antenna previously conditioned). They always responded significantly more to the ipsilateral than to the contralateral antenna. higher than to the contralateral mechanosensory stimulation (empty bar; $F_{1,81}=288 ; P<0.00001$ ), thus showing that sidespecificity of mechanosensory memory was also maintained after a differential conditioning procedure.

\section{DISCUSSION}

The present work introduces a form of associative conditioning in honeybees that seems to be dominated by Pavlovian components as it is based on an elemental association between mechanosensory, antennal stimulation and a reward of sucrose solution delivered to the proboscis. This form of learning has been reported before (Giurfa et al. 2001; Menzel et al. 2001) but it was never characterized with respect to its properties. Here we show that bees can learn an association between side-specific mechanosensory stimulation and sucrose solution and that responses produced by the paired training have a true associative basis. Responding is only elicited by mechanosensory stimulation and not by spurious cues such as olfactory, visual, and contextual ones. The interstimulus interval (ISI) affected one-trial mechanosensory learning: a bell-shaped curve with a maximum of responses at $4 \mathrm{sec}$ ISI was obtained. Thus, $24 \mathrm{~h}$ after conditioning, mechanosensory memory is still operative. Beyond absolute, side-specific, mechanosensory conditioning, differential, sidespecific, mechanosensory conditioning is also possible.

Our paradigm contains essentially Pavlovian components and differs from the antennal learning introduced by Erber and coworkers $(1997,1998)$ because, in our case, reward delivery was not dependent on the bees' behavior. Kisch and Erber (1999) have shown that their antennal learning paradigm, in which bees learn to vary their frequency of antennal contacts with a plate presented close to their head, has a true operant basis. In this case, the yoked control procedure yielded no acquisition, thus showing that their antennal learning was operant. In the procedures in which bees learn either the contour of an object close to their antennae or to discriminate different textures by scanning them with the antennae and associating them with the presence or absence of reward (Erber et al. 1998), it is the active movement of the antennae that allows such discrimination. In our case, the movement of the antennae is not essential for reward delivery as the contingency established is one between mechanosensory stimulation, decided by the experimenter (and not by the bee), and a reward of sucrose. Moreover, Erber et al. (1998) state that the discrimination of tactile surfaces is abolished when the antennal tip is covered by paint; in our case, the tip of the antennae rarely contacted the toothpick because the experimenter stimulated the antennae by moving them at the level of the scapus and joint between scapus and flagellum (i.e., close to the basis of the antennae). This difference in the antennal areas involved in the processing of conditioned stimuli is consistent with the idea that mechanosensory receptors in Erber's and our paradigm are differently localized on the antenna.

A remarkable feature of our mechanosensory-conditioning paradigm is that it yields results that are extremely similar to those obtained in olfactory classical conditioning of honeybees (Bitterman et al. 1983; Menzel et al. 1993). The dynamics of acquisition in absolute and differential conditioning, the curve showing the effect of ISI on one-trial learning, the results of the omission procedure, and the yoke control are comparable in both paradigms (see, for instance, Bitterman et al. 1983; Menzel et al. 1993). Memory dynamics show also some similarities as mechanosensory memory is intact $24 \mathrm{~h}$ after the last conditioning trials. Considering these similarities, it would be worth testing retrieval $3 \mathrm{~min}$ after side-specific mechanosensory conditioning to determine whether a decay in retrieval is also observed in the case of mechanosensory learning, indicating a transfer be- 
tween short-term and medium-term memory phases as in the case of olfactory learning (Menzel et al. 1993). Furthermore, it would be worth studying the potential of backward pairings to induce inhibitory learning, as shown by Hellstern et al. (1998) for olfactory learning.

We have excluded the possibility that bees use olfactory information in our experiments. Thus, a possible explanation for the similarities between olfactory and mechanosensory conditioning is the relative coincidence of the respective neural pathway of these two sensory modalities (Arnold et al. 1985; Maronde 1991). Olfactory receptors are distributed along the antenna, whereas mechanosensory receptors can also be found at several locations on the antenna (Pareto 1972; Suzuki 1975). Olfactory receptors send their projections via the antennal nerve to the antennal lobe, the primary olfactory neuropile of the bee brain; each antennal lobe is constituted by 160 glomeruli (Flanagan and Mercer 1989a; Galizia et al. 1999), which represent the convergence sites of olfactory receptor neurons onto projection neurons that send their axons to higher-order centers in the brain (mushroom bodies and lateral horn; Abel et al. 2001), but also onto local, inhibitory interneurons (Gascuel and Masson 1991; Fonta et al. 1993). Mechanoreceptors, on the other hand, project to the dorsal lobe, a brain structure associated with motor centers (Suzuki 1975; Homberg et al. 1989; Maronde 1991; Kloppenburg 1995). Flanagan and Mercer (1989b) have shown a complete class of interneurons, which interconnect the dorsal lobe and the antennal lobe. These neurons target the T4 region of the antennal lobe. Thus, at the level of the output of the antennal lobe, olfactory and mechanosensory information might be integrated into a common across-fiber activity pattern. This suggestion is consistent with the reports showing that projection neurons respond in some cases to mechanosensory and olfactory stimuli (for review, see Homberg et al. 1989). The antennal lobe could be, therefore, a likely integration center for olfactory and mechanosensory processing. Optical imaging techniques currently available for the study of olfactory coding at the level of the antennal lobe (Joerges et al. 1997) could be useful to determine whether or not mechanosensory stimulations as used here elicit specific glomerular activation patterns similar to those generated by olfactory stimulations (Joerges et al. 1997; Galizia and Menzel 2000).

Such a sensory integration is certainly present at the level of higher-order brain structures such as the mushroom bodies. Mobbs (1982) and Strausfeld (2002) stated that the basal ring of the mushroom bodies receives, besides olfactory input, mechanosensory input, although they did not define the neural origin of such input. A mushroom body extrinsic neuron, the PE1 neuron (Mauelshagen 1993), which connects the peduncle of the mushroom body with two areas of the protocerebrum in the honeybee brain, the lateral protocerebral lobe (LPL) and the ring neuropile around the $\alpha$-lobe, responds to mechanosensory stimulation, thus suggesting that mechanosensory pathways should reach the mushroom bodies (Rybak and Menzel 1998).

For the elemental, unimodal associative task under study, however, the mushroom bodies may not be of fundamental importance. Recently, it has been suggested that for such elemental, unimodal, nonambiguous tasks (i.e., a conditioned stimulus is unambiguously associated with reinforcement or with absence of it), the complete mushroom bodies may not be necessary (Scheiner et al. 2001b; Malun et al. 2002; see, however, Cano Lozano et al. 2001) as learning-associated changes in neural representations can be found already at the level of the antennal lobe in olfactory conditioning (Faber et al. 1999). Moreover, elemental differential olfactory conditioning can be achieved by bees having partial side-specific mushroom body lesions generated by treatment with hydroxyurea at the larval stage (Malun et al. 2002). In this case, conditioning the antenna corresponding to the side of the brain in which the mushroom bodies are ablated yields similar results to those obtained when conditioning the intact side of the same bees or either side of nonablated, control bees. Similarly, in the case of the operant conditioning of antennal movements (Kisch and Erber 1999), the response of a single motoneuron from the dorsal lobe can account for the behavioral plasticity found in this paradigm (Erber et al. 2000), thus underlining the fact that mushroom bodies are not necessarily involved in elemental, unimodal associative learning tasks.

An intriguing question raised by our results relates to the real nature of olfactory classical conditioning in honeybees. The conventional procedure used for such conditioning associates odor delivery with a reward of sucrose delivered to the antenna, usually by touching it with a capillary or a toothpick imbibed with sucrose solution, and then to the extended proboscis. Sucrose delivery to the antenna elicits proboscis extension reflex but implies also a mechanosensory component. To which extent olfactory conditioning of such reflex is a composite bimodal conditioning (olfactory + mechanosensory) remains unanswered. In our case, sucrose was always delivered to the proboscis and never to the antennae. Note that olfactory conditioning is also possible if sucrose is only delivered to the proboscis (Bitterman et al. 1983; Sandoz et al. 2002).

Our paradigm is certainly perfectible. In particular, fundamental improvements can be achieved at the level of the delivery and control of the conditioned stimulus. In our experiments, mechanosensory stimulation was provided by gentle contact of the antenna with a toothpick. This approach does not allow controlling the intensity of the conditioned stimulus, a feature that is of fundamental importance in classical conditioning experiments. This is due to the fact that CS intensity depends here on the subjective perception of the experimenter. To correct this aspect, a current goal in our laboratory is to achieve mechanosensory conditioning by using automatically controlled procedures for antennal stimulation. As a conclusion, we characterize here a new learning paradigm that exploits the fact that bees can learn to associate a mechanosensory antennal stimulation with a reinforcement of sucrose solution. Studying this paradigm appropriately, as we did, constitutes an important step toward its implementation as a standard procedure for further learning studies in honeybees.

\section{MATERIALS AND METHODS}

Honeybees (Apis mellifera L.) were caught at the entrance of outdoor hives at the beginning of each experimental day. Each bee was placed in a small glass vial and cooled in a freezer until it ceased its movements. Individuals were mounted into restraining harnesses such that they could only move their antennae and mouth parts, including the proboscis (Bitterman et al. 1983). Animals were then kept undisturbed in a room of the laboratory for $\sim 3-4 \mathrm{~h}$. Then, $15 \mathrm{~min}$ before starting the experiments, each subject was checked for intact proboscis extension reflex by lightly touching one antenna with a toothpick imbibed with sucrose solution without subsequent feeding. Extension of the proboscis beyond the virtual line between the open mandibles was counted as PER (unconditioned reaction). Animals that did not show the reflex $(<5 \%)$ were not used in the experiments.

The onset and offset of each trial as well as of conditioned and unconditioned stimulus delivery were controlled and signaled by a computer that was programmed to emit tones of different frequencies for each event. At the beginning of each trial, the subject was placed in the experimental site for $27 \mathrm{sec}$ to allow familiarization with the training situation. Thereafter, the conditioned stimulus (CS) was presented for $4 \mathrm{sec}$. It consisted of a mechanosensory stimulation provided by touching one antenna with a clean toothpick. The toothpick was replaced by a new one in each assay and from bee to bee. To avoid uncontrolled visual stimulation (i.e., visual information provided by a sudden ap- 
proach of the toothpick to the antenna), the toothpick was placed always close to the antenna. Only during CS delivery did the toothpick contact the antenna. In all but the fifth experiment, the onset of the unconditioned stimulus (US) occurred 3 sec after CS onset ("forward pairing") in reinforced trials and lasted $3 \mathrm{sec}$. The US was always $1.25 \mathrm{M}$ sucrose solution delivered to the proboscis by means of another toothpick imbibed with the sucrose solution. Contact of the sucrose solution with the mouth parts alone was sufficient to elicit PER (Bitterman et al. 1983) because of the abundant presence of sucrose receptors on the proboscis and adjacent buccal pieces (Whitehead and Larsen 1976). The US was never delivered to the antenna, to avoid confounded mechanosensory stimulation. After proboscis extension, the bee was allowed to feed for $3 \mathrm{sec}$. Therefore, the interstimulus interval (ISI) was $3 \mathrm{sec}$, and the overlap between CS and US was $1 \mathrm{sec}$. After US offset, the bee was left undisturbed in the experimental site for $27 \mathrm{sec}$ and then returned to its resting position to allow the next bee to be conditioned. The total time in the experimental site was thus $1 \mathrm{~min}$ for each bee. Nonreinforced trials consisted of 4-sec CS presentation without reward. The intertrial interval was always $10 \mathrm{~min}$ (CS+ to CS+ interval).

During acquisition we recorded whether a bee extended its proboscis after onset of the mechanosensory stimulation (CS) and before presentation of the sucrose solution (US; conditioned reaction). Multiple PER responses during a CS were counted as a single PER. After experiments were finished, all animals were again checked for proboscis extension reflex. If an animal did not respond, it was discarded $(<10 \%)$.

\section{Statistics}

Repeated-measurement analysis of variance ANOVA was used for between-group as well as within-group comparisons. Although parametric analysis of variance is usually not allowed in case of dichotomous data such as those of the PER, Monte Carlo studies have shown that it is permissible to use ANOVAs for dichotomous dependent variables under certain conditions, which are met by our data: equal cell frequencies and at least 40 degrees of freedom of the error term (Lunney 1970). Where necessary, Newman-Keuls tests modified for repeated measurements were used to perform post hoc comparisons. The $\alpha$-level was set to 0.05 (two-tailed) for all analyses.

\section{ACKNOWLEDGMENTS}

We thank Matthieu Dacher, Monique Gauthier, Jean-Christophe Sandoz, and two anonymous referees for useful comments and corrections on the manuscript. We thank Tilman Franke for Figure 1. This work was supported by the SFB 515 (Special Research Area of the German Research Council) and by the program Action Cognitique (French Research Ministry) and the Institut Universitaire de France. We especially acknowledge the valuable help of Mareike Becker, Elodie Constans, Marianne Latinus, Valerie Marty, Patricia Nierle, and Vanina Vergoz with some experiments.

The publication costs of this article were defrayed in part by payment of page charges. This article must therefore be hereby marked "advertisement" in accordance with 18 USC section 1734 solely to indicate this fact.

\section{REFERENCES}

Abel, R., Rybak, J., and Menzel, R. 2001. Structure and response pattern of olfactory interneurons in the honeybee, Apis mellifera. J. Comp. Neurol. 437: 363-383.

Arnold, G., Masson, C., and Budharugsa, S. 1985. Comparative study of the antennal lobes and their afferent pathway in the worker bee and the drone (Apis mellifera). Cell Tissue Res. 242: 593-605.

Bitterman, M.E., Menzel, R., Fietz, A., and Schäfer, S. 1983. Classical conditioning of proboscis extension in honeybees (Apis mellifera). J. Comp. Psychol. 97: 107-119.

Brembs, B. and Heisenberg, M. 2000. The operant and the classical in conditioned orientation of Drosophila melanogaster at the flight simulator. Learn. Mem. 7: 104-115.

Cano Lozano, V., Armengaud, C., and Gauthier, M. 2001. Memory impairment induced by cholinergic antagonists injected into the mushroom bodies of the honeybee. J. Comp. Physiol. A 187: $249-254$

Chandra, S. and Smith, B.H. 1998. An analysis of synthetic processing of odor mixtures in the honeybee (Apis mellifera). J. Exp. Biol. 201: $3113-3121$.

Deisig, N., Lachnit, H., Giurfa, M., and Hellstern, F. 2001. Configural olfactory learning in honeybees: Negative and positive patterning discrimination. Learn. Mem. 8: 70-78.

Deisig, N., Lachnit, H., and Giurfa, M. 2002. The effect of similarity between elemental stimuli and compounds in olfactory patterning discriminations. Learn. Mem. 9: 112-121.

Deisig, N., Lachnit, H., Sandoz, J.-C., Lober, K., and Giurfa, M. 2003. A modified version of the unique cue theory accounts for olfactory compound processing in honeybees. Learn. Mem. 10: 199-208.

Erber, J., Pribbenow, B., Grandy, K., and Kierzek, S. 1997. Tactile motor learning in the antennal system of the honeybee (Apis mellifera L.). J. Comp. Physiol. A 181: 355-365.

Erber, J., Kierzek, S., Sander, E., and Grandy, K. 1998. Tactile learning in the honeybee. J. Comp. Physiol. A 183: 737-744.

Erber, J., Pribbenow, B., Kisch, J., and Faensen, D. 2000. Operant conditioning of antennal muscle activity in the honey bee (Apis mellifera L.). J. Comp. Physiol. A 186: 557-565.

Faber, T., Joerges, J., and Menzel, R. 1999. Associative learning modifies neural representations of odors in the insect brain. Nat. Neurosci. 2: 74-78.

Flanagan, D. and Mercer, A.R. 1989a. An atlas and 3-D reconstruction of the antennal lobes in the worker honey bee Apis mellifera L. Hymenoptera: Apidae. Int. J. Insect Morphol. Embryol. 18: 145-159. . 1989b. Morphology and response characteristics of neurones in the deutocerebrum of the brain in the honeybee Apis mellifera. J. Comp. Physiol. A 164: 483-494.

Fonta, C., Sun, X.J., and Masson, C. 1993. Morphology and spatial distribution of bee antennal lobe interneurones responsive to odours. Chem. Senses 18: 101-119.

Galizia, C.G. and Menzel, R. 2000. Odour perception in honeybees: Coding information in glomerular patterns. Curr. Opin. Neurobiol. 10: $504-510$.

Galizia, C.G., McIllwright, S., and Menzel, R. 1999. A digital three-dimensional atlas of the honeybee antennal lobe based on optical sections acquired by confocal microscopy. Cell Tissue Res. 295: 383-394.

Gascuel, J. and Masson, C. 1991. A quantitative ultrastructural study of the honeybee antennal lobe. Tissue Cell 23: 341-355.

Gerber, B. and Smith, B.H. 1998. Visual modulation of olfactory learning in honeybees. J. Exp. Biol. 201: 2213-2217.

Giurfa, M. 2003a. The amazing mini brain: Lessons from a honeybee. Beeworld 84: 5-18.

. 2003b. Cognitive neuroethology: Dissecting non-elemental learning in a honeybee brain. Curr. Opin. Neurobiol. 13: 726-735.

Giurfa, M., Nierle, P., Becker, M., and Malun, D. 2001. Mechanosensory classical conditioning in the honeybee. In Proceedings of the 4th Meeting German Neuroscience Society-28th Göttingen Neurobiology Conference II. p. 684. Thieme, Stuttgart-New York.

Hammer, M. and Menzel, R. 1995. Learning and memory in the honeybee. J. Neurosci. 15: 1617-1630.

Hellstern, F., Malaka, R., and Hammer, M. 1998. Backward inhibitory learning in honeybees: A behavioral analysis of reinforcement processing. Learn. Mem. 4: 429-444.

Homberg, U., Christensen, T.A., and Hildebrand, J.G. 1989. Structure and function of the deutocerebum in insects. Annu. Rev. Entomol. 34: 477-501.

Joerges, J., Küttner, A., Galizia, C.G., and Menzel, R. 1997. Representations of odors and odor mixtures visualized in the honeybee brain. Nature 387: 285-288

Kisch, J. and Erber, J. 1999. Operant conditioning of antennal movements in the honey bee. Behav. Brain Res. 99: 93-102.

Kloppenburg, P. 1995. Anatomy of the antennal motoneurons in the brain of the honeybee (Apis mellifera). J. Comp. Neurol. 363: 333-343.

Lunney, G.H. 1970. Using analysis of variance with a dichotomous dependent variable: An empirical study. J. Educat. Meas. 7: 263-269.

Malun, D., Giurfa, M., Galizia, C.G., Plath, N., Brandt, R., Gerber, B. and Eisermann, B. 2002. Hydroxyurea-induced partial mushroom body ablation does not affect acquisition and retention of olfactory differential conditioning in honeybees J. Neurobiol. 53: 343-360.

Maronde, U. 1991. Common projection areas of antennal and visual pathways in the honeybee brain, Apis mellifera. J. Comp. Neurol. 309: 328-340.

Masuhr, T. and Menzel, R. 1972. Learning experiments on the use of sidespecific information in the olfactory and visual system in the honeybee (Apis mellifica). In Information processing in the visual systems of arthropods (ed. R. Wehner), pp. 315-322. Springer, Berlin-Heidelberg-New York. 
Mauelshagen, J. 1993. Neural correlates of olfactory learning in an identified neuron in the honey bee brain. J. Neurophysiol. 69: 609-625.

Menzel, R. 1999. Memory dynamics in the honeybee. J. Comp. Physiol. A 185: 323-340.

Menzel, R. and Bitterman, M.E. 1983. Learning by honey bees in an unnatural situation. In Behavioral physiology and neuro-ethology: Roots and growing points (eds. F. Huber and H. Markl), pp. 206-215. Springer, Berlin-Heidelberg-New York.

Menzel, R. and Giurfa, M. 2001. Cognitive architecture of a minibrain: The honeybee. Trends Cogn. Sci. 5: 62-71.

Menzel, R. and Müller, U. 1996. Learning and memory in honeybees: From behavior to neural substrates. Annu. Rev. Neurosci. 19: 379-404.

Menzel, R., Greggers, U., and Hammer, M. 1993. Functional organization of appetitive learning and memory in a generalist pollinator, the honeybee. In Insect learning: Ecological and evolutionary perspectives (eds. D. Papaj and A.C. Lewis), pp. 79-125. Chapman and Hall, New York.

Menzel, R., Manz, G., Menzel, R.M., and Greggers, U. 2001. Massed and spaced learning in honeybees: The role of CS, US, the inter-trial interval and the test interval. Learn. Mem. 8: 198-208.

Mobbs, P.G. 1982. The brain of the honeybee Apis mellifera I. The connections and spatial organization of the mushroom bodies. Philos. Trans. R. Soc. Lond. B Biol. Sci. 298: 309-354.

Pareto, A. 1972. Die zentrale Verteilung der Fühlerafferenz bei Arbeiterinnen der Honigbiene, Apis mellifera L. Z. Zellforsch. Mikrosc. Anat. 131: 485-509.

Pavlov, I. 1927. Conditioned reflexes. Oxford University Press, Oxford, UK.

Pelz, C., Gerber, B., and Menzel, R. 1997. Odorant intensity as a determinant for olfactory conditioning in honeybees: Roles in discrimination, overshadowing and memory consolidation. J. Exp. Biol. 200: 837-847.

Pribbenow, B. and Erber, J. 1996. Modulation of antennal scanning in the honeybee by sucrose stimuli, serotonin and octopamine: Behavior and electrophysiology. Neurobiol. Learn. Mem. 66: 109-120.

Rybak, J. and Menzel, R. 1998. Integrative properties of the Pe1 neuron, a unique mushroom body output neuron. Learn Mem. 5: 133-145.
Sandoz, J.-C. and Menzel, R. 2001. Side-specificity of olfactory learning in the honeybee: Generalization between odors and sides. Learn. Mem. 8: 286-294.

Sandoz, J.-C., Hammer, M., and Menzel, R. 2002. Side-specificity of olfactory learning in the honeybee: US input side. Learn. Mem. 9: $337-348$.

Sandoz, J.-C., Galizia, G., and Menzel, R. 2003. Side-specific olfactory conditioning leads to more specific odor representation between sides but not within sides in the honeybee antennal lobes. Neuroscience 120: 1137-1148.

Scheiner, R., Erber, J., and Page Jr., R.E. 1999. Tactile learning and the individual evaluation of the reward in honey bees (Apis mellifera L.). J. Comp. Physiol. A 185: 1-10.

Scheiner, R., Page Jr., R.E., and Erber, J. 2001a. The effects of genotype, foraging role, and sucrose responsiveness on the tactile learning performance of honey bees (Apis mellifera L.). Learn. Mem. 76: $138-150$.

Scheiner, R., Weiß, A., Malun, D., and Erber, J. 2001b. Learning in honey bees with brain lesions: How partial mushroom-body ablations affect sucrose responsiveness and tactile antennal learning. Anim. Cognit. 4: 227-235.

Skinner, B. 1938. The behavior of organisms. Appleton, New York.

Strausfeld, N.J. 2002. Organization of the honey bee mushroom body: Representation of the calyx within the vertical and $\gamma$ lobes. J. Comp. Neurol. 450: 4-33.

Suzuki, H. 1975. Antennal movements induced by odour and central projection of the antennal neurons in the honeybee. J. Insect Physiol. 21: 831-847.

Takeda, K. 1961. Classical conditioned response in the honey bee. J. Insect Physiol. 6: 168-179.

Whitehead, A.T. and Larsen, J.R. 1976. Electrophysiological responses of galeal contact chemoreceptors of Apis mellifera to selected sugars and electrolytes. J. Insect Physiol. 22: 1609-1616.

Received May 30, 2003; accepted in revised form February 11, 2004.

\section{Learning \& Memory}




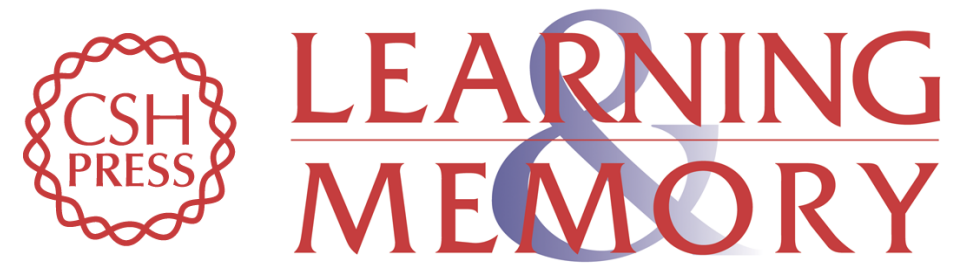

\section{Associative Mechanosensory Conditioning of the Proboscis Extension Reflex in Honeybees}

Martin Giurfa and Dagmar Malun

Learn. Mem. 2004, 11:

Access the most recent version at doi:10.1101/lm.63604

References This article cites 46 articles, 13 of which can be accessed free at:

http://learnmem.cshlp.org/content/11/3/294.full.html\#ref-list-1

License

Email Alerting Receive free email alerts when new articles cite this article - sign up in the box at the Service top right corner of the article or click here. 